\title{
Effects of color superconductivity on the nucleation of quark matter in neutron stars
}

\author{
I. Bombaci ${ }^{1}$, G. Lugones ${ }^{1}$, and I. Vidaña ${ }^{2}$ \\ 1 Dipartimento di Fisica “Enrico Fermi” Università di Pisa and INFN Sezione di Pisa, Largo Bruno Pontecorvo 3, 56127 Pisa, Italy \\ e-mail: [bombaci;lugones]@df.unipi.it \\ 2 Departament d'Estructura i Constituents de la Materia, Universitat de Barcelona, Avda. Diagonal 647, 08028 Barcelona, Spain \\ e-mail: vidana@ecm.ub.es
}

Received 23 March 2006 / Accepted 27 September 2006

\begin{abstract}
Aims. We study the nucleation of quark matter drops at the center of cold deleptonized neutron stars. These drops can be made up of unpaired quark matter or of color-superconducting quark matter, depending on the details of the equations of state for quark and hadronic matter. The nature of the nucleated phase is relevant in determining the critical mass $M_{\mathrm{cr}}$ of hadronic stars above which a transition to a quark star (strange or hybrid) is possible.

Methods. We investigate the dependence of $M_{\text {cr }}$ upon the parameters of the quark model (the Bag constant $B$, the pairing gap $\Delta$, and the surface tension $\sigma$ of the quark-hadron interface) and for different parametrizations of the hadronic equation of state. We also calculate the energy released when a pure hadronic star with the critical mass is converted into a quark star.

Results. In general, the dependence of $M_{\mathrm{cr}}$ on $B, \Delta$, and $\sigma$ is mild if the parameters of the quark model correspond to hybrid stars, and strong if they correspond to strange stars. Also, the critical mass always decreases with $\Delta$ and increases with $B$ and $\sigma$. The total released energy is in the range $3 \times 10^{52}-4 \times 10^{53} \mathrm{erg}$.

Conclusions. For a large part of the parameter space corresponding to hybrid stars, the critical mass is very close to (but smaller than) the maximum mass of hadronic stars, so compatible with a "mixed" population of compact stars (pure hadronic up to the critical mass and hybrid above the critical mass). For very large $B$ the critical mass is never lower than the maximum mass of hadronic stars, implying that quark stars cannot form through the mechanism studied here. The energy released in the conversion is sufficient for powering a gamma ray burst.
\end{abstract}

Key words. elementary particles - stars: neutron - equation of state - gamma rays: bursts

\section{Introduction}

The nucleation of quark matter in neutron stars has been studied by many authors, due to the potential connection with explosive astrophysical events such as supernovae and gamma ray bursts. Some of the earlier studies of quark-matter nucleation (see e.g. Horvath et al. 1992; Horvath 1994; Olesen \& Madsen 1994; and references therein) dealt with thermal nucleation in hot and dense hadronic matter. In these studies, it was found that the prompt formation of a critical-size drop of quark matter via thermal activation is possible above a temperature of about 2-3 MeV. As a consequence, it was inferred that pure hadronic stars are converted to strange stars or to hybrid stars within the first seconds after their birth. However, neutrino trapping in the protoneutron star phase strongly precludes the formation of a quark phase (Lugones \& Benvenuto 1998; Benvenuto \& Lugones 1999; Vidaña et al. 2005). Therefore, it is possible that the compact star survives the early stages of its evolution as a pure hadronic star. In this case, nucleation of quark matter would be triggered by quantum fluctuations in degenerate $(T=0)$ neutrino-free hadronic matter (see e.g. Grassi 1998; Iida \& Sato 1998; Berezhiani et al. 2003; Harko et al. 2004; Bombaci et al. 2004, and references therein).

A general feature of degenerate Fermi systems is that they become unstable if any attractive interaction exists at the Fermi surface (Bardeen et al. 1957). This instability leads to the formation of a condensate of Cooper pairs and the appearance of superconductivity. In QCD any attractive quark-quark interaction will lead to pairing and color superconductivity (Barrois 1977; Bailin \& Love 1984, and references therein). Since the typical superconducting gaps in quark matter may be as large as $\Delta \sim 100 \mathrm{MeV}$ (see e.g. Alford 2001, and references therein), it is interesting to study the effects of color superconductivity in the process of nucleation. Some recent studies have gone in this direction (Drago et al. 2004; Lugones \& Bombaci 2005), but our study here is more complete and self-consistent.

In a recent work (Lugones \& Bombaci 2005, hereafter LB05), we have studied the formation of superconducting quark matter in bulk, paying particular attention to the microscopic state of quark matter just after the deconfinement. As explained in LB05, several states should be possible. For example, quantum fluctuations could form a drop of $\beta$-stable quark matter (hereafter the $Q^{\beta}$ phase). However, this is strongly suppressed with respect to the formation of a non $\beta$-stable drop by a factor $\sim G_{\mathrm{Fermi}}^{2 N / 3}$, where $N$ is the number of particles in the criticalsize quark drop. This is so because the formation of a $\beta$-stable drop would involve the almost simultaneous conversion of $\sim N / 3$ up and down quarks into strange quarks. For a critical-size $\beta$ stable nugget at the center of a neutron star $N \sim 100-1000$ is found, and therefore the factor is actually tiny. This is the same reason that impedes an iron nucleus converting into a drop of strange quark matter, even in the case in which strange quark 
matter has a lower energy per baryon (Bodmer-Witten-Terazawa hypothesis).

However, quantum fluctuations can form the so called $Q^{*}$ phase bubbles, in which the flavor content of the quark phase is equal to that of the $\beta$-stable hadronic system at the same pressure. Since no flavor conversion is involved, there are no suppressing Fermi factors, and a $Q^{*}$-phase drop can be nucleated much more easily. Once a critical-size drop of the $Q^{*}$-phase is formed, the weak interactions will have enough time to act, changing the quark flavor fraction of the deconfined droplet to lower its energy, and a droplet of the ( $\beta$-stable) $Q^{\beta}$-phase is formed. Notice that in degenerate matter, the intermediate phase can be made up of unpaired quark matter (hereafter the $Q_{\mathrm{unp}}^{*}$ phase) or by color-superconducting quark matter $\left(Q_{\Delta}^{*}\right.$ phase). Which one of the two kinds of droplets ( $Q_{\text {unp }}^{*}$ or $Q_{\Delta}^{*}$ ) will nucleate depends on the Gibbs free energy per baryon of each phase ( $\left.g_{\text {unp }}, g_{\Delta}\right)$, as discussed in detail in LB05.

The analysis in LB05 was made in bulk, i.e. without taking into account the energy cost due to finite size effects in creating a drop of deconfined quark matter in the hadronic environment. As a consequence of the surface effects, it is necessary to have an overpressure $\Delta P=P-P_{0}$ with respect to the bulk transition point $P_{0}$ (see Fig. 1 in LB05). Thus, above $P_{0}$, hadronic matter is in a metastable state, and the formation of a real drop of quark matter occurs via a quantum nucleation mechanism. In this work we study the quantum nucleation process including the effect of color superconductivity, and analyze the implications for neutron stars ${ }^{1}$.

A final comment is worthwhile concerning the formation of mixed hadron-quark phases, in which the electric charge is zero globally but not locally, i.e. the two phases have opposite charges (Glendenning 1992, 2001). As discussed in Lugones \& Benvenuto (1998), mixed phases cannot form in the here studied just-deconfined phase, because the flavor conservation condition guarantees that a just-deconfined quark-matter drop initially has exactly the same electric charge as the hadronic drop from which it originated (i.e. zero). Of course, charge separation could occur later on (if energetically preferred) and a mixed phase could form. However, notice that Debye screening effects and the surface tension can prevent mixed phases to form (see e.g. Tatsumi \& Voskresensky 2003; Endo et al. 2005; Maruyama et al. 2006, and references therein). In any case, this study of mixed phases concerns the state of the system at times that are much longer than the ones that are addressed in this paper.

\section{The properties of the intermediate non- $\beta$-stable quark phase}

As emphasized in the Introduction the intermediate phase can be in an unpaired state $\left(Q_{\text {unp }}^{*}\right)$ or in the so-called two-flavor color superconducting (2SC) state (hereafter $Q_{\Delta}^{*}$ ). In this section we resume the equation of state of the $Q_{\Delta}^{*}$-phase (for more details see LB05).

The physical conditions imposed on the $Q_{\Delta}^{*}$-phase are flavor conservation and color neutrality of the quark gas. Flavor conservation means that the particle number (per baryon) of quarks $u$, $d$ and $s$ are the same in the hadronic phase and in the $Q_{\Delta}^{*}$-phase. The only difference is that quarks are confined inside hadrons in

1 It is worth mentioning that Drago et al. (2004) have investigated the nucleation of a superconducting $\beta$-stable phase made up by colorflavor-locked (CFL) quark matter. However, as stated before, the direct nucleation of a drop of such a phase is discouraged by weak interactions. the hadronic phase, and in the $Q_{\Delta}^{*}$-phase they are deconfined and paired. This condition can be expressed in terms of two parameters $\xi$ and $\eta$ :

$n_{\mathrm{d}}=\xi n_{\mathrm{u}}$

$n_{\mathrm{s}}=\eta n_{\mathrm{u}}$

where $n_{i}$ is the particle number density of the $i$-species in the quark phase. The quantities $\xi \equiv Y_{\mathrm{d}}^{\mathrm{H}} / Y_{\mathrm{u}}^{\mathrm{H}}$ and $\eta \equiv Y_{\mathrm{s}}^{\mathrm{H}} / Y_{\mathrm{u}}^{\mathrm{H}}$ are functions of the pressure, and they characterize the composition of the hadronic phase. These expressions are valid for any hadronic EOS. For hadronic matter containing $n, p, \Lambda, \Sigma^{+}, \Sigma^{0}$, $\Sigma^{-}, \Xi^{-}$, and $\Xi^{0}$, we have

$\xi=\frac{n_{\mathrm{p}}+2 n_{\mathrm{n}}+n_{\Lambda}+n_{\Sigma^{0}}+2 n_{\Sigma^{-}}+n_{\Xi^{-}}}{2 n_{\mathrm{p}}+n_{\mathrm{n}}+n_{\Lambda}+2 n_{\Sigma^{+}}+n_{\Sigma^{0}}+n_{\Xi^{0}}}$
$\eta=\frac{n_{\Lambda}+n_{\Sigma^{+}}+n_{\Sigma^{0}}+n_{\Sigma^{-}}+2 n_{\Xi^{0}}+2 n_{\Xi^{-}}}{2 n_{\mathrm{p}}+n_{\mathrm{n}}+n_{\Lambda}+2 n_{\Sigma^{+}}+n_{\Sigma^{0}}+n_{\Xi^{0}}}$.

Notice that $\xi$ and $\eta$ univocally determine the number of electrons present in the system through electric charge neutrality of the deconfined phase:

$3 n_{\mathrm{e}}=2 n_{\mathrm{u}}-n_{\mathrm{d}}-n_{\mathrm{s}}$.

We also impose two pairing conditions, $n_{\mathrm{dr}}=n_{\mathrm{ug}}$ in order to allow quarks $d_{\mathrm{r}}$ to be paired with $u_{\mathrm{g}}$ and $n_{\mathrm{ur}}=n_{\mathrm{dg}}$ in order to allow quarks $u_{\mathrm{r}}$ to be paired with $d_{\mathrm{g}}$. Finally, the system must be globally colorless, that is, $n_{\mathrm{r}}=n_{\mathrm{g}}=n_{\mathrm{b}}$.

From the above equations we obtain the number densities of each quark species in the paired phase as functions of the flavor composition $\eta, \xi$ :

$\begin{aligned} n_{\mathrm{ub}} & =\frac{4-2 \xi}{1+\xi} n_{\mathrm{ur}} \\ n_{\mathrm{db}} & =\frac{-2+4 \xi}{1+\xi} n_{\mathrm{ur}} \\ n_{\mathrm{sb}} & =\frac{2 \eta}{1+\xi} n_{\mathrm{ur}} \\ n_{\mathrm{e}} & =\frac{2(2-\eta-\xi)}{1+\xi} n_{\mathrm{ur}}\end{aligned}$

The other particle densities are given by $n_{\mathrm{ug}}=n_{\mathrm{dr}}=n_{\mathrm{dg}}=n_{\mathrm{ur}}$ and $n_{\mathrm{sg}}=n_{\mathrm{sr}}=n_{\mathrm{sb}}$.

The pressure and Gibbs energy per baryon of the paired deconfined phase can also be written in terms of the same parameters:

$$
\begin{aligned}
P_{\left(Q *_{\Delta}\right)} & =\sum_{\mathrm{fc}} \frac{k_{\mathrm{fc}}^{4}}{12 \pi^{2}}+\frac{\mu_{\mathrm{e}}^{4}}{12 \pi^{2}}+\frac{1}{\pi^{2}} \bar{\mu}^{2} \Delta^{2}-B, \\
\mu_{\left(Q *_{\Delta}\right)} & =\sum_{\mathrm{fc}} \frac{n_{\mathrm{fc}} \mu_{\mathrm{fc}}}{n_{\mathrm{B}}}+\frac{\mu_{\mathrm{e}} n_{\mathrm{e}}}{n_{\mathrm{B}}}, \\
n_{\mathrm{B},\left(Q *_{\Delta}\right)} & =\sum_{\mathrm{fc}} n_{\mathrm{fc}},
\end{aligned}
$$

where $k_{\mathrm{fc}}=\left(\mu_{\mathrm{fc}}^{2}-m_{\mathrm{fc}}^{2}\right)^{1 / 2}$ and $\bar{\mu}=\mu_{\mathrm{ur}}$. The chemical potentials $\mu_{\mathrm{fc}}$ are obtained by inverting the following set of equations,

$$
\begin{aligned}
n_{\mathrm{fc}}=\frac{\mu_{\mathrm{fc}}^{3}}{3 \pi^{2}}+\frac{2 \Delta^{2} \bar{\mu}}{\pi^{2}} & f_{\mathrm{c}}=u_{\mathrm{r}}, u_{\mathrm{g}}, d_{\mathrm{r}}, d_{\mathrm{g}} \\
\mu_{\mathrm{fc}}=\left(3 \pi^{2} n_{\mathrm{fc}}\right)^{1 / 3} & f_{\mathrm{c}}=u_{\mathrm{b}}, d_{\mathrm{b}} \\
\mu_{\mathrm{fc}}=\left[\left(3 \pi^{2} n_{\mathrm{fc}}\right)^{2 / 3}+m_{\mathrm{s}}^{2}\right]^{1 / 2} & f_{\mathrm{c}}=s_{\mathrm{r}}, s_{\mathrm{g}}, s_{\mathrm{b}}
\end{aligned}
$$

where the number densities are given by Eqs. (6)-(9). 
For sufficiently high values of $\Delta$ the energy cost invested in forcing the 2SC pairing pattern is compensated by the gain in the condensation energy, and the preferred phase is $Q_{\Delta}^{*}$. For low values of $\Delta$ the preferred state is the $Q_{\text {unp }}^{*}$-phase described in Olesen \& Madsen (1994), Lugones \& Benvenuto (1998), and Bombaci et al. (2004).

\section{Quantum nucleation of quark matter bubbles}

To calculate the nucleation rate of quark matter in the hadronic medium we use the Lifshitz-Kagan quantum nucleation theory (Lifshitz \& Kagan 1972) in the relativistic form given by Iida $\&$ Sato (1998). The QM droplet is supposed to be a sphere of radius $\mathcal{R}$ and its quantum fluctuations are described by the Lagrangian

$L(\mathcal{R}, \dot{\mathcal{R}})=-\mathcal{M}(\mathcal{R}) c^{2} \sqrt{1-(\dot{\mathcal{R}} / c)^{2}}+\mathcal{M}(\mathcal{R}) c^{2}-U(\mathcal{R})$,

where $\mathcal{M}(\mathcal{R})$ is the effective mass of the QM droplet, and $U(\mathcal{R})$ its potential energy.

Within the Lifshitz-Kagan quantum nucleation theory, one assumes that the phase boundary (i.e. the droplet surface) moves slowly compared to the high sound velocity of the medium $\left(\dot{\mathcal{R}} \ll v_{\mathrm{s}} \sim c\right.$ ). Thus the number density of each phase adjusts adiabatically to the fluctuations of the droplet radius, and the system retains pressure equilibrium between the two phases. Thus, the droplet's effective mass is given by (Lifshitz \& Kagan 1972; Iida \& Sato 1998)

$\mathcal{M}(\mathcal{R})=4 \pi \rho_{\mathrm{H}}\left(1-\frac{n_{\mathrm{b}, Q *}}{n_{\mathrm{b}, \mathrm{H}}}\right)^{2} \mathcal{R}^{3}$,

where $\rho_{\mathrm{H}}$ is the hadronic mass density, $n_{\mathrm{b}, \mathrm{H}}$ and $n_{\mathrm{b}, Q *}$ are the baryonic number densities at a same pressure in the hadronic and $Q^{*}$-phase, respectively. The difference in the Gibbs free energy is given by (Lifshitz \& Kagan 1972; Iida \& Sato 1998)

$U(\mathcal{R})=\frac{4}{3} \pi \mathcal{R}^{3} n_{\mathrm{b}, Q *}\left(\mu_{Q *}-\mu_{\mathrm{H}}\right)+4 \pi \sigma \mathcal{R}^{2}$,

where $\mu_{\mathrm{H}}$ and $\mu_{Q *}$ are the hadronic and quark chemical potentials at a fixed pressure $P$. For comparison with previous works notice that $\mu$ is the same as the bulk Gibbs energy per baryon $g=(\rho+$ $P) / n_{\mathrm{B}}=\left(\sum_{i} n_{i} \mu_{i}\right) / n_{\mathrm{B}}$. For $\sigma=0$ we have $U(\mathcal{R})=$ Volume $\times n_{\mathrm{B}} \times$ $\Delta g$, and the bulk limit is recovered.

We neglected the term associated with the curvature energy, and also terms connected with the electrostatic energy, since they are known to introduce small corrections (Iida \& Sato 1998; Bombaci et al. 2004). The value of the surface tension $\sigma$ for the interface separating the quarks and hadrons phases is poorly known, and typical values used in the literature range within 10-50 MeV/fm ${ }^{2}$ (Heiselberg et al. 1993; Iida \& Sato 1998). Notice that, since we are considering $\sigma$ as a parameter, color superconductivity enters the above expressions only through $\mu_{Q *}$ and $n_{\mathrm{b}, Q *}$ (see next section).

The probability of forming a bubble with a critical radius can be computed using a semiclassical approximation. The procedure is rather straightforward. First one uses the well known Wentzel-Kramers-Brillouin (WKB) approximation to compute the ground state energy $E_{0}$ and the oscillation frequency $v_{0}$ of the virtual QM drop in the potential well $U(\mathcal{R})$. Then it is possible to calculate the probability of tunneling in a relativistic framework as (Iida \& Sato 1998)

$p_{0}=\exp \left[-\frac{A\left(E_{0}\right)}{\hbar}\right]$ where $A$ is the action under the potential barrier

$A(E)=\frac{2}{c} \int_{\mathcal{R}_{-}}^{\mathcal{R}_{+}}\left\{\left[2 \mathcal{M} c^{2}+E-U\right][U-E]\right\}^{1 / 2} \mathrm{~d} \mathcal{R}$,

$\mathcal{R}_{ \pm}$being the classical turning points.

The nucleation time is then equal to

$\tau=\left(v_{0} p_{0} N_{\mathrm{c}}\right)^{-1}$,

where $N_{\mathrm{c}}$ is the number of virtual centers of droplet formation in the innermost region of the star. Following the simple estimate given in Iida \& Sato (1998), we take $N_{\mathrm{c}}=10^{48}$. The uncertainty in the value of $N_{\mathrm{c}}$ is expected to be within one or two orders of magnitude. In any case, all the qualitative features of our scenario will be not affected by the uncertainty in the value of $N_{\mathrm{c}}$.

In order to explore the astrophysical implications of the nucleation, we introduce the concept of critical mass for a hadronic star (see e.g. Bombaci et al. 2004). In bulk, the transition begins when the Gibbs conditions $\Delta P=0$ and $\Delta \mu=0$ are verified. However, as a consequence of the surface effects, it is now necessary to have an overpressure $\Delta P=P-P_{0}>0$ with respect to the bulk transition point. This overpressure will produce $\Delta \mu<0$ in Eq. (18) allowing the barrier to be tunneled. Since there is an univocal relation between the mass of a compact star and its central pressure, we can consider that the nucleation time in Eq. (21) is a function of the mass of the star. The higher the overpressure, the easier to nucleate a bubble. In other words, the higher the mass of a pure hadronic star, the shorter the time to nucleate a quark drop at the center of the star. We define as critical mass $M_{\text {cr }}$ of the metastable hadronic star (HS) the value of the gravitational mass for which the nucleation time is equal to one year: $M_{\mathrm{cr}} \equiv M_{\mathrm{HS}}(\tau=1 \mathrm{yr})$. It is worth recalling that the nucleation time given in Eq. (21) is an extremely steep function of the hadronic star mass (see e.g. Bombaci et al. 2004). Therefore, the exact value of the nucleation time ( $\tau=1 \mathrm{yr}$ in our case) chosen in the definition of $M_{\mathrm{cr}}$ is not relevant ${ }^{2}$. For example, a variation of several orders of magnitude in the choice of $\tau$ (e.g. $1 \mathrm{~s}$ or $10^{17} \mathrm{~s}$ ) will change $M_{\mathrm{cr}}$ by less than $0.1 \%$. Therefore, pure hadronic stars with $M_{\mathrm{HS}}>M_{\mathrm{cr}}$ are very unlikely to be observed, while pure hadronic stars with $M_{\mathrm{HS}}<M_{\mathrm{cr}}$ are safe with respect to a sudden transition to quark matter. Then $M_{\mathrm{cr}}$ plays the role of an effective maximum mass for the hadronic branch of compact stars (see the discussion in Bombaci et al. 2004). While the Oppenheimer-Volkov maximum mass $M_{\mathrm{HS}}^{\max }$ (Oppenheimer \& Volkov 1939) is determined by the overall stiffness of the EOS for hadronic matter, the value of $M_{\mathrm{cr}}$ will depend in addition on the properties of the intermediate non- $\beta$ stable quark phase ( $Q_{\mathrm{unp}}^{*}$ or $Q_{\Delta}^{*}$ ). As emphasized in LB05, the critical mass exists even in the absence of surface effects, although its value is lower.

The results are shown in Figs. 1-4 where we show the critical mass of hadronic stars as a function of the different parameters of the equations of state. We have adopted rather common models for describing both the hadronic and the quark phases of dense matter. For the hadronic phase we used models that are based on a relativistic Lagrangian of hadrons interacting via the exchange of sigma, rho, and omega mesons. The parameters adopted are the standard ones (Glendenning \& Moszkowski 1991). Hereafter we refer to this model as the GM equation of

\footnotetext{
2 Notice also that, due to this tremendously steep dependence of $\tau$ on the mass of the star, the poor knowledge of the factor $N_{\mathrm{c}}$ in Eq. (21) does not modify the value obtained for $M_{\mathrm{cr}}$ significantly.
} 

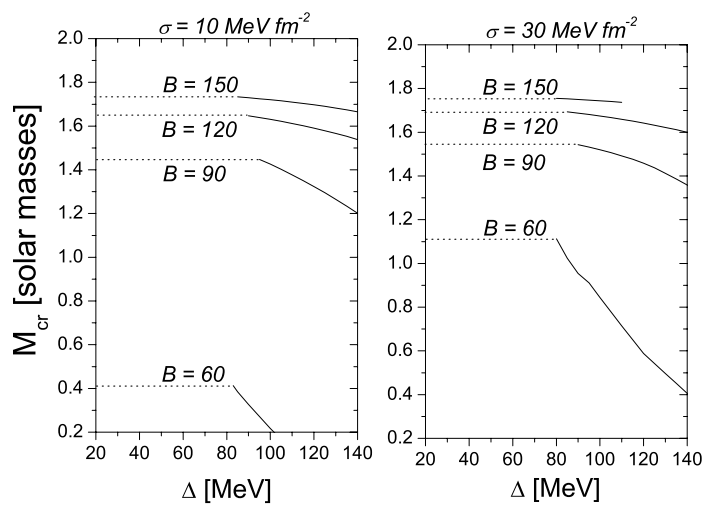

Fig. 1. The critical mass of hadronic stars for the nucleation of a quark matter drop at the center. We have considered the parametrization GM1 (Glendenning \& Moszkowski 1991) for the EOS of the hadronic phase, and two different values for the surface tension of the quark-hadron interface: $\sigma=10 \mathrm{MeV} / \mathrm{fm}^{2}$ and $\sigma=30 \mathrm{MeV} / \mathrm{fm}^{2}$. Different curves correspond to different values of the Bag constant (indicated in units of $\mathrm{MeV} \mathrm{fm}^{-3}$ ). For low values of the pairing gap $\Delta$ the nucleated intermediate phase is unpaired $\left(Q_{\mathrm{unp}}^{*}\right)$ and is indicated with a dotted line. For sufficiently high $\Delta$ the preferred intermediate phase is paired $\left(Q_{\Delta}^{*}\right)$ and the critical mass decreases substantially (solid line). The maximum mass for hadronic stars with the GM1 EOS is $M_{\mathrm{HS}}^{\max }=1.78 M_{\odot}$.
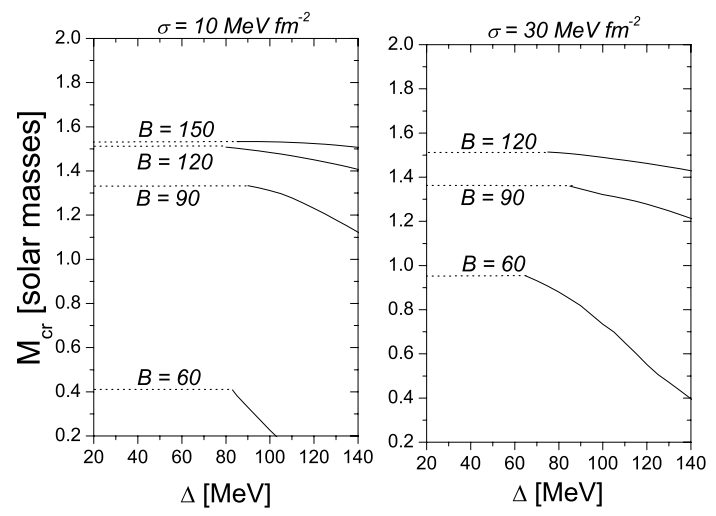

Fig. 2. The same as Fig. 1 but using GM3 for the hadronic phase. The maximum mass for hadronic stars with the GM3 EOS is $M_{\mathrm{HS}}^{\max }=$ $1.53 M_{\odot}$.

state $(\mathrm{EOS})^{3}$. For quark matter we used the MIT Bag model ${ }^{4}$ with $m_{\mathrm{u}}=m_{\mathrm{d}}=0, m_{\mathrm{s}}=150 \mathrm{MeV}$, and $\alpha_{\mathrm{s}}=0$.

Before analyzing the results, it is worthwhile pointing out that there are two qualitatively different possibilities concerning the state of quark matter: either it is absolutely stable (i.e. it has an energy per baryon at $P=0$ and $T=0$ that is lower than the mass of the neutron) or it is not absolutely stable. Since the actual case in nature is unknown, the so-called stability windows have been introduced (Farhi \& Jaffe 1984). They show the regions in which each possibility is realized as a function of the

3 The names GM1 and GM3 refer to the parameters given in the first and third lines of Table 2 of Glendenning \& Moszkowski (1991). The corresponding compressibility and coupling constants are the following. For GM1: $K=300 \mathrm{MeV},\left(g_{\sigma} / m_{\sigma}\right)^{2}=11.79 \mathrm{fm}^{2},\left(g_{\omega} / m_{\omega}\right)^{2}=$ $7.149 \mathrm{fm}^{2},\left(g_{\rho} / m_{\rho}\right)^{2}=4.411 \mathrm{fm}^{2}, b=0.002947, c=-0.001070$. For GM3: $K=240 \mathrm{MeV},\left(g_{\sigma} / m_{\sigma}\right)^{2}=9.927 \mathrm{fm}^{2},\left(g_{\omega} / m_{\omega}\right)^{2}=4.820 \mathrm{fm}^{2}$, $\left(g_{\rho} / m_{\rho}\right)^{2}=4.791 \mathrm{fm}^{2}, b=0.008659, c=-0.002421$. The two hadronic models include the lowest baryon octet $\left(n, p, \Lambda, \Sigma^{+}, \Sigma^{0}, \Sigma^{-}, \Xi^{-}\right.$, and $\left.\Xi^{0}\right)$.

${ }^{4}$ Other models, such as the Nambu - Jona - Lasinio model (see Buballa 2005), can give different results, specially in the low-density regime for which the chiral symmetry is not completely restored.
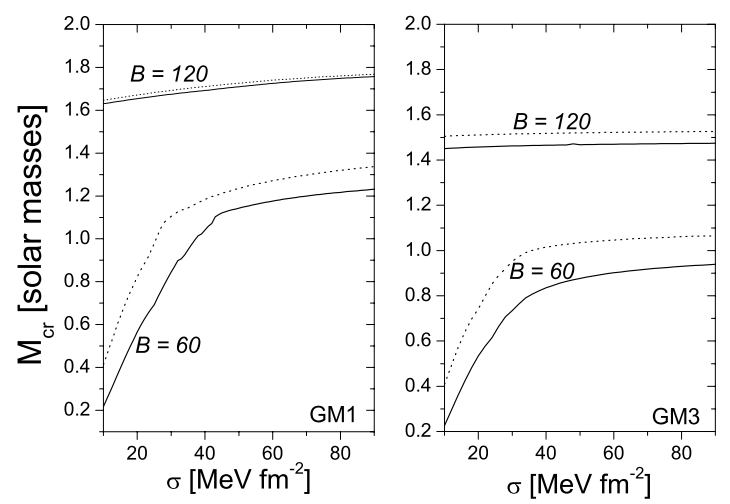

Fig. 3. The critical mass of hadronic stars as a function of the surface tension $\sigma$ of the quark-hadron interphase. We show the results for two values of the Bag constant $B$ (indicated in units of $\mathrm{MeV} \mathrm{fm}^{-3}$ ). Dotted lines correspond to unpaired quark matter, solid lines to paired quark matter with $\Delta=100 \mathrm{MeV}$.
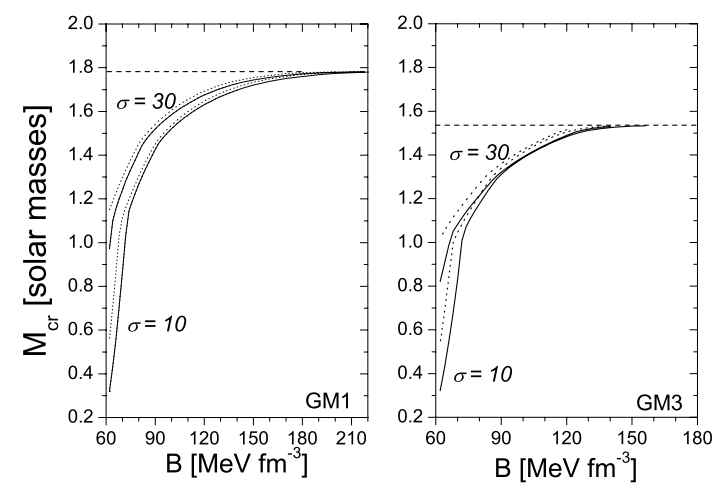

Fig. 4. The critical mass of hadronic stars as a function of the Bag constant $B$. The results are shown for $\sigma=10$ and $30 \mathrm{MeV} \mathrm{fm}^{-2}$. Dotted lines correspond to unpaired quark matter, solid lines to paired quark matter with $\Delta=100 \mathrm{MeV}$. The dashed horizontal line indicates the maximum mass of hadronic stars for each hadronic equation of state.

different parameters of the quark model (e.g. the strange quark mass $m_{\mathrm{s}}$, the Bag constant $B$, and the pairing gap $\Delta$ ). The stability windows for color-flavor locked quark matter have been presented in Lugones \& Horvath (2002). Fixing the value of the strange quark mass to $m_{\mathrm{s}}=150 \mathrm{MeV}$, it is found that paired $\beta$-stable quark matter is absolutely stable for

$B<73.1 \mathrm{MeV} \mathrm{fm}^{-3}\left[1+0.53\left(\frac{\Delta}{100 \mathrm{MeV}}\right)^{2}\right]$

The above expression is an approximation to order $m_{\mathrm{s}}^{2}$, accurate within a few percent in the range of interest (see Eq. (17) of Lugones \& Horvath 2002). If quark matter is not absolutely stable, the stars containing quark matter are hybrid stars, i.e. ones containing $\beta$-stable quark matter only at their interiors. If quark matter is absolutely stable, all the stars containing quark matter are strange stars, i.e. made up of $\beta$-stable quark matter from the center to the surface.

In Figs. 1 and 2 the effect of the pairing gap $\Delta$ is seen clearly. For low values of $\Delta$, the nucleated intermediate phase is in an unpaired state. For sufficiently high values of $\Delta$ the gain of the condensation energy favors the nucleation of a paired state $\left(Q_{\Delta}^{*}\right)$ and the critical mass decreases substantially due to the lower energy cost of the phase. Notice that the effect of pairing is stronger for low values of the Bag constant $B$, where it can change $M_{\mathrm{cr}}$ by more than a factor of 2 . On the other hand, the variation in 
the critical mass due only to pairing effects (i.e. at constant $B$ ) is smaller than $\sim 20 \%$ in the regime of large $B$ that corresponds to hybrid stars (i.e. non absolute stability of quark matter).

In Fig. 3 we see the effect of the surface tension $\sigma$ on the critical mass $M_{\text {cr }}$. The effect of $\sigma$ is small for $B$ larger than $\sim 100 \mathrm{MeV} \mathrm{fm}^{-3}$. Therefore, in this regime of $B$ the bulk limit is a good approximation (cf. LB05). For $B$ smaller than $\sim 100 \mathrm{MeV} \mathrm{fm}^{-3}$ the effects of $\sigma$ are important, and they increase their relevance as $B$ decreases. This can be seen in Fig. 3 in the case for $B=60 \mathrm{MeV} \mathrm{fm}^{-3}$, where $M_{\mathrm{cr}}$ changes by a factor of 4 in the most plausible range for $\sigma$ (between 10 and $30 \mathrm{MeV} \mathrm{fm}^{-2}$ ). However, notice that the curves tend to "saturate" for higher $\sigma$.

In Fig. 4 we explore the dependence of the results on the Bag constant $B$. We can identify three qualitatively different regions:

(1) Region of Large $B$ : for $B$ larger than a critical value ( $\sim 200 \mathrm{MeV} \mathrm{fm}^{-3}$ for GM1 and $\sim 150 \mathrm{MeV} \mathrm{fm}^{-3}$ for GM3) the critical mass $M_{\mathrm{cr}}$ is no longer lower than the maximum mass for hadronic stars $M_{\mathrm{HS}}^{\max }$. This implies that quark stars cannot form by means of quantum nucleation in this region of the parameter space.

(2) Region of intermediate B corresponding to hybrid stars: for $B$ between $\sim 100 \mathrm{MeV} \mathrm{fm}^{-3}$ and $\sim 150-200 \mathrm{MeV} \mathrm{fm}^{-3}$, the critical mass $M_{\text {cr }}$ is very close (but lower than) the maximum mass of hadronic stars. Notice that for this range of $B$, stars containing quark matter are hybrid since quark matter in $\beta$ equilibrium has lower energy per baryon (at $P=0$ ) than the neutron mass (we assume for simplicity that $\beta$-stable quark matter is in a color-flavor locked state).

(3) Region of $B$ corresponding to strange stars: for sufficiently small $B$, quark matter in $\beta$-equilibrium is absolutely stable (see Eq. (22)). As is apparent from Fig. 4, the critical mass $M_{\text {cr }}$ strongly depends on $B$ in this case.

\section{Energy released in the conversion}

The amount of energy that can be released in the conversion of a pure hadronic star (HS) into a strange star or a hybrid star has been calculated in several previous works (Bombaci \& Datta 2000; Berezhiani et al. 2003; Bombaci et al. 2004; Drago et al. 2004). Moreover, Drago et al. (2004) calculated the available energy including the effect of color superconductivity in the final star. However, since the critical masses in that work were not calculated with the intermediate non- $\beta$-stable phase, we present new self-consistent results here.

As in previous works, we define $\Delta E$ as the difference between the gravitational mass of the initial hadronic star and that of the final (hybrid or strange) star having the same baryonic mass:

$\Delta E=\left[M_{G}(H S)-M_{G}(Q S)\right] c^{2}$.

According to the definition, $\Delta E$ is the total energy that can be released in the conversion. This energy can be liberated in an explosive manner (and give rise to GRBs) or in a less violent way (in which case observable signals would be less spectacular), but this depends on complex processes that are beyond the scope of the present work (see e.g. Lugones et al. 2002; Ouyed et al. 2005; Paczynski \& Haensel 2005; Bhattacharyya et al. 2006, and references therein).

The results are shown in Figs. 5 and 6 where we show $\Delta E$ as a function of the pairing gap $\Delta$ for different values of the bag constant $B$, using the equations of state GM1 and GM3 for hadronic matter, and assuming that the $\beta$-stable quark phase (for the final stellar configuration) is in the color-flavor-locked (CFL) state.

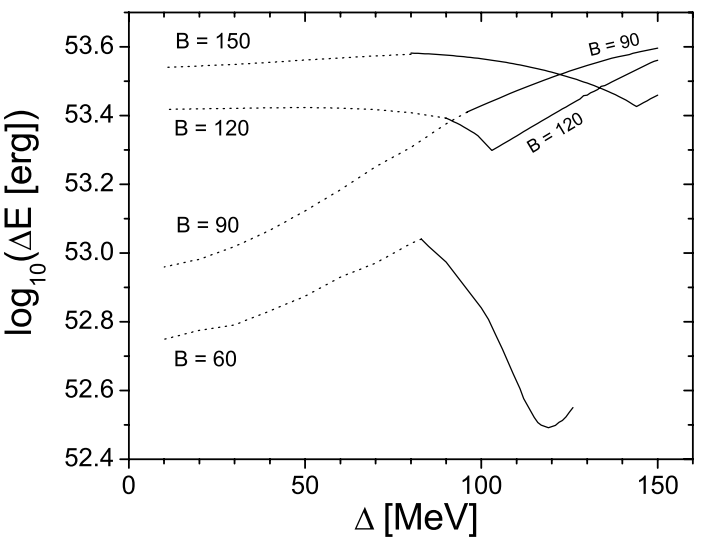

Fig. 5. The total energy released in the conversion of a hadronic star (with the critical mass) into a quark star (hybrid star or strange star depending on the values of $B$ and $\Delta$ ). The dotted lines show the cases where the intermediate non- $\beta$-stable quark phase is unpaired, while the full lines correspond to the case where the intermediate phase is paired (see more details in the text). In this figure we used the GM1 EOS for hadronic matter. The critical mass is calculated with $\sigma=10 \mathrm{MeV} \mathrm{fm}^{-2}$. The Bag constant is indicated in units of $\mathrm{MeV} \mathrm{fm}^{-3}$.

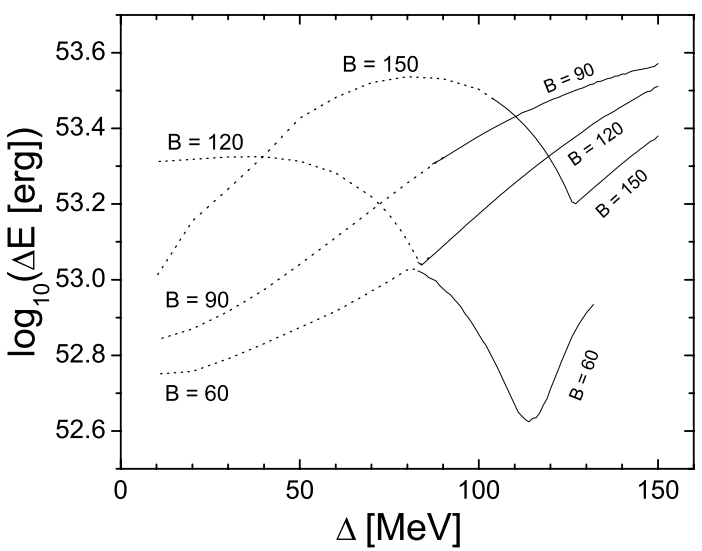

Fig. 6. The same as Fig. 5 but employing the GM3 EOS for hadronic matter.

We assume that a given hadronic star can convert into a quark star provided it has the critical mass. Those with a lower mass cannot convert, and those with higher mass are already converted. Each point on the curves of Figs. 5 and 6 indicate the energy released in the conversion of a hadronic star having the critical mass corresponding to $B$ and $\Delta$. This hadronic star is converted into a quark star (with the same barionic mass) that can be a strange star or a hybrid star also depending on the values of $B$ and $\Delta$ (known with the help of Eq. (22)).

The released energy is mainly the result of two competing effects. For $\Delta$ above a threshold value (see Figs. 1 and 2), pairing tends to diminish $M_{\mathrm{cr}}$ and, therefore, decreases the released energy since the conversion happens at the critical mass. On the other hand, there is an effect on the gravitational mass of the hybrid or strange star due to the condensation term in the equation of state of CFL quark matter. Depending on $B$ and $\Delta$, the equation of state can be stiffer or softer than the unpaired case (see e.g. Lugones \& Horvath 2003) and therefore $\Delta E$ can be either an increasing or a decreasing function of $\Delta$.

The dotted part of the curves in Figs. 5 and 6 corresponds to the case in which the intermediate non- $\beta$-stable quark phase is unpaired. Therefore, the critical mass is independent of $\Delta$ and the variations in $\Delta E$ are only due to the variation in the 
stiffness of the equations of state that changes the gravitational mass of the final star. The full-line part of the curves corresponds to the case in which the intermediate phase is paired. Since the critical mass is a decreasing function of $\Delta$ (see Figs. 1 and 2), the released energy tends to decrease, specially for low values of $B$, for which the decrease in $M_{\mathrm{cr}}$ is stronger (see e.g. the case of $B=60 \mathrm{MeV} \mathrm{fm}^{-3}$ ). For larger $B$ the decrease in $M_{\mathrm{cr}}$ is not enough to compensate for the effect of the stiffness of the equations of state. In fact, for very large $\Delta, \Delta E$ is always an increasing function of $\Delta$ because the gain in the condensation energy compensates for all other effects.

\section{Discussion and conclusions}

In the present work we have studied the nucleation of quark matter drops at the center of cold deleptonized neutron stars. These drops can be made up of unpaired quark matter or by color-superconducting quark matter. The nature of the nucleated phase depends on the details of the equations of state for quark and hadronic matter and is relevant in the determination of the critical mass $M_{\text {cr }}$ of the hadronic stars above which a transition to quark matter is possible. We calculated $M_{\mathrm{cr}}$ as a function of the several parameters of the quark model and for different parametrizations of the hadronic equations of state. In general, the dependence of $M_{\mathrm{cr}}$ on $B, \Delta$, and $\sigma$ is mild if the parameters of the quark model correspond to hybrid stars, and strong if they correspond to strange stars. Also, the critical mass always decreases with $\Delta$ and increases with $B$ and $\sigma$.

As stated before, if quark matter is not absolutely stable, the stars containing quark matter are hybrid stars, i.e. containing $\beta$-stable quark matter only at their interiors. On the other hand, if quark matter is absolutely stable, all the stars containing quark matter are strange stars, i.e. those made up of $\beta$-stable quark matter from the center up to the surface. Moreover, it has been argued that if $\beta$-stable quark matter is absolutely stable, then all neutron stars should be made up of quarks. The reason is that cosmic ray strangelets will be created after the merging of a strange star with another compact star in a binary system. The rate of binary mergers in the galaxy appears to be high enough so that even a conservative estimate gives a large galactic production rate of strangelets $\left(10^{-10} M_{\odot} / \mathrm{yr}\right)$ and, correspondingly, a large flux in the interstellar medium. When a strangelet penetrates a neutron star, it will grow by absorbing free neutrons, thereby converting the whole star into strange quark matter. Similarly, all massive stars that accumulate a strangelet in its core will give birth to strange stars rather than neutron stars after the explosion as a core-collapse supernova. Because of these arguments, it has been argued that, if some neutron stars are undoubtedly identified as conventional, the inevitable conclusion is that strange quark matter is not absolutely stable and only conventional neutron stars or (eventually) hybrid stars exist (see Caldwell \& Friedman 1991; Balberg 2005). However, notice that the strangelet contamination of the galaxy may be suppressed, for example, if strange quark stars are discouraged in binaries (Belczynski et al. 2002, and references therein). On the other hand, strangelets could disintegrate when impacting onto the external layers of the star. But, the physics of strangelet fragmentation is largely unknown, and it is not clear whether it is effective for all incident energies (especially for those strangelets impacting with low energy). In conclusion, if quark matter is in fact absolutely stable, it is not clear whether the conversion would be triggered from inside (quantum nucleation) or from outside (strangelet contamination), and therefore, it is uncertain whether the critical mass studied in this paper is meaningful in the corresponding region of the parameter space $(B, \Delta$, etc.).

On the other hand, if quark matter is not absolutely stable, strangelets do not exist. Therefore, the trigger of the conversion to quark matter cannot come from outside the neutron star, and the mechanism studied here is the most plausible one for triggering the conversion within cold deleleptonized neutron stars. In this context, a relevant result presented here is that for a large region of the parameter space corresponding to hybrid stars, the critical mass is very near to (but smaller than) the maximum mass of hadronic stars. This means that all compact stars with masses up to $M_{\text {cr }}$ should be normal hadronic stars, while those with masses above $M_{\text {cr }}$ may be hybrid stars (if they do not collapse into a black hole). In the rest of the parameter space corresponding to hybrid stars, the critical mass $M_{\mathrm{cr}}$ is no longer lower than the maximum mass for hadronic stars $M_{\mathrm{HS}}^{\max }$. This implies that quark stars cannot form by means of quantum nucleation in this region of the parameter space, and probably they don't exist at all (if other mechanism of quark matter formation does not operate).

We have also calculated the amount of energy that can be released in the conversion of a hadronic star into a quark or hybrid star (assuming that the $\beta$-stable quark matter phase is in a color-flavor-locked state). The total released energy is in the range $3 \times 10^{52}-4 \times 10^{53} \mathrm{erg}$, therefore it is sufficient for powering a gamma ray burst.

\section{References}

Alford, M. G. 2001, Ann. Rev. Nucl. Part. Sci., 51, 131

Bardeen, J., Cooper, L. N., \& Schrieffer, J. R. 1957, Phys. Rev., 108, 1175

Barrois, B. 1977, Nucl. Phys. B, 129, 390

Bailin, D., \& Love, A. 1984, Phys. Rep., 107, 325

Bhattacharyya, A., Ghosh, S. K., Raha, S. 2006, Phys. Lett. B, 635, 195

Baym, G., \& Chin, S. A. 1976, Phys. Lett. B, 62, 241

Benvenuto, O. G., \& Lugones, G. 1999, MNRAS, 304, L25

Balberg, S. 2004, Phys. Rev. Lett., 92, 119001

Berezhiani, Z., Bombaci, I., Drago, A., Frontera, F., \& Lavagno, A. 2003, ApJ, 586,1250

Bombaci, I., \& Datta, B. 2000, ApJ, 530, L69

Bombaci, I., Parenti, I., \& Vidaña, I. 2004, ApJ, 614, 314

Buballa, M. 2005, Phys. Rep., 407, 205

Caldwell, R. R., \& Friedman, J. L. 1991, Phys. Lett. B, 264, 143

Drago, A., Lavagno, A., \& Pagliara, G. 2004, Phys. Rev. D, 69, 057505

Drago, A., Lavagno, A., \& Parenti, I. 2005 [arXiv:astro-ph/0512652]

Endo, T., Maruyama, T., Chiba, S., \& Tatsumi, T. 2006, Progr. Theor. Phys., 115, 337

Farhi, E., \& Jaffe, R. L. 1984, Phys. Rev. D, 30, 2379

Grassi, F. 1998, ApJ, 492, 263

Glendenning, N. K. 1992, Phys. Rev. D, 46, 1274

Glendenning, N. K. 2001, Phys. Rep., 342, 393

Glendenning, N. K., \& Moszkowski, S. A. 1991, Phys. Rev. Lett., 67, 2414

Harko, T., Cheng, K. S., \& Tang, P. S. 2004, ApJ, 608, 945

Horvath, J. E. 1994, Phys. Rev. D, 49, 5590

Horvath, J. E., Benvenuto, O. G., \& Vucetich, H. 1992, Phys. Rev. D, 45, 3865

Iida, K., \& Sato, K. 1998, Phys. Rev. C, 58, 2538

Lifshitz, I. M., \& Kagan, Y. 1972, Sov. Phys. JETP, 35, 206

Lugones, G., \& Benvenuto, O. G. 1998, Phys. Rev. D, 58, 083001

Lugones, G., \& Horvath, H. 2002, Phys. Rev. D, 66, 074017 (2005)

Lugones, G., \& Horvath, H. 2003, A\&A, 403, 173

Lugones, G., \& Bombaci, I. 2005, Phys. Rev. D, 72, 065021 (LB05)

Lugones, G., Ghezzi, C. R., de Gouveia Dal Pino, E. M., \& Horvath, J. E. 2002, ApJ, 581, L101

Maruyama, T., Tanigawa, T., Chiba, S., et al. 2006, Proceedings of Science JHW2005, 024

Olesen, M. L., \& Madsen, J. 1994, Phys. Rev. D, 49, 2698

Ouyed, R., Rapp, R., \& Vogt, C. 2005, ApJ, 632, 1001

Paczynski, B., \& Haensel, P. 2005, MNRAS, 362, L4

Tatsumi, T., \& Voskresensky, D. N. 2003, [nucl-th/0312114]

Vidaña, I., Bombaci, I., \& Parenti I. 2005, J. Phys. G, 31, S1165 